\title{
A Condition Number for Multifold Conic Systems
}

\author{
Dennis Cheung \\ United International College \\ Tang Jia Wan \\ Zhuhai, Guandong Province \\ P.R. of CHINA \\ e-mail: dennisc@uic.edu.hk \\ Felipe Cucker* \\ Department of Mathematics \\ City University of Hong Kong \\ 83 Tat Chee Avenue, Kowloon \\ $\mathrm{HONG} \mathrm{KONG}$ \\ e-mail: macucker@math.cityu.edu.hk \\ Javier Peña \\ Tepper School of Business \\ Carnegie Mellon University \\ 5000 Forbes Avenue, Pittsburgh, PA 15213-3890 \\ USA \\ e-mail: jfp@andrew.cmu.edu
}

\begin{abstract}
The analysis of iterative algorithms solving a conic feasibility problem $A y \in K$, with $A$ a linear map and $K$ a regular, closed, convex cone, can be conveniently done in terms of Renegar's condition number $C(A)$ of the input data $A$. In this paper we define and characterize a condition number which exploits the possible factorization of $K$ as a product of simpler cones. This condition number, which extends the one defined in [Math. Program., 91:163-174, 2001] for polyhedral conic systems, captures better the conditioning of the problem by filtering out, e.g., differences in scaling between components corresponding to different factors of $K$. We see these results as a step in developing a theory of conditioning that takes into account the structure of the problem.
\end{abstract}

*Partially supported by CityU SRG grant \#7001860 


\section{Introduction}

\subsection{Multifold Conic Systems and Condition}

Let $X, Y$ be real, finite dimensional, Banach spaces, and $K \subseteq X$, a regular closed convex cone (a precise definition is in Section 2 below). Denote by $L(Y, X)$ the space of linear maps from $Y$ to $X$ endowed with the operator norm. Given $A \in L(Y, X)$ consider the feasibility problem: decide whether there exists $y \in Y$ satisfying

$$
A y \in K
$$

This format encompasses, after homogenization, a large class of feasibility problems. For example, the linear programming feasibility problem corresponds to $K=\mathbb{R}_{+}^{n}$, the positive orthant in $\mathbb{R}^{n}$, and semidefinite programming corresponds to $K=\mathbf{S}_{+}^{n}$, the set of $n \times n$ positive semi-definite matrices. Consider also the alternative feasibility problem

$$
A^{*} x^{*}=0, x \in K^{*}
$$

where $X^{*}, Y^{*}$ are the dual spaces of $X, Y$ respectively, $A^{*} \in L\left(X^{*}, Y^{*}\right)$ is the adjoint of $A$, and $K^{*} \subseteq X^{*}$ is the dual cone of $K$.

The problem (1) is strictly feasible if there exists $y \in Y$ such that $A y \in$ $\operatorname{int}(K)$. Let $\mathcal{D}$ denote the set of instances $A \in L(Y, X)$ for which (1) is strictly feasible. Observe that $A \in \mathcal{D}$ if and only if $A Y-K=X$, i.e., if and only if the conic system

$$
A y-c \in K
$$

is feasible for every $c \in X$. In addition, for the set $\mathcal{P}:=\overline{\mathcal{D}}^{c}$ (where the bar denotes topological closure and the ${ }^{c}$ complement) it follows that $A \in \mathcal{P}$ if and only if $A^{*} K^{*}=Y^{*}$. That is, if for all $b^{*} \in Y^{*}$ the system

$$
A^{*} x^{*}=b^{*}, x^{*} \in K^{*}
$$

is feasible. It is also the case that $A \in \mathcal{P}$ if and only if $A^{*}$ is surjective and the problem (2) is strictly feasible, i.e., there exists $x^{*} \in \operatorname{int}\left(K^{*}\right)$ such that $A^{*} x^{*}=0$.

The sets $\mathcal{D}$ and $\mathcal{P}$ are the set of "well-posed" feasible instances for problems (1) and (2) respectively. The boundary $\Sigma:=\partial \mathcal{D}=\partial \mathcal{P}$ is the set of "ill-posed" instances. Given $A \in \Sigma$, arbitrarily small perturbations of $A$ may yield instances both in $\mathcal{D}$ and $\mathcal{P}$.

The feasibility problems (1) and (2) can be solved via iterative algorithms (such as interior-point, or ellipsoid methods). The running time of such 
algorithms grows as $A$ approaches $\Sigma$. Consequently, complexity analysis of these algorithms could be carried out in terms of a measure capturing this distance. ${ }^{1}$ A general analysis of such type for interior-point methods is due to Renegar [24, 25], who introduced the condition number

$$
C(A)=\frac{\|A\|}{\operatorname{dist}(A, \Sigma)}=\frac{\|A\|}{\min _{\widetilde{A} \in \Sigma}\|\widetilde{A}-A\|} .
$$

Renegar's condition number is thus the normalized inverse of the distance to ill-posedness. The condition number $C(A)$ can also be used in the complexity analysis of the ellipsoid method [17], and in the round-off analysis of interior-point algorithms [11]. For the linear programming feasibility problem $\left(K=\mathbb{R}_{+}^{n}\right)$, the quantities $C(A)$ and $\ln C(A)$ have also been analysed as random variables when $A$ is random $[7,14]$. Bounds for the expected value of $C(A)$ (or for that of $\ln C(A)$ ) yield average case bounds for the algorithms mentioned above.

It is often the case that a feasibility problem of the form (1) is actually the coupling of a number of similar feasibility problems. More precisely, if $X=X_{1} \times \cdots \times X_{r}$ and $K=K_{1} \times \cdots \times K_{r}$ where each $K_{j} \subseteq X_{j}$ is a regular closed convex cone, then (1) can be written as

$$
\begin{aligned}
A_{1} y & \in K_{1} \\
& \vdots \\
A_{r} y & \in K_{r},
\end{aligned}
$$

where each $A_{j} \in L\left(Y, X_{j}\right)$ is the $j$ th projection of $A \in L(Y, X)$ onto $L\left(Y, X_{j}\right)$. In this multifold case, it may well be the case that $C(A)$ is large without (6) being intrinsically poorly conditioned. This could happen, e.g., if the feasibility components $A_{j} y \in K_{j}$ are scaled differently. In such case a natural preconditioning of (6), such as component normalization, would remove the seemingly bad-conditioning. In the case of linear programming (i.e., when $X=\mathbb{R}^{n}, K=\mathbb{R}_{+}^{n}, r=n$, and $K_{j}=\mathbb{R}_{+}$) another condition number, $\mathscr{C}(A)$, was introduced in [6] (extending ideas in [19]) exploiting the multifold structure of $\mathbb{R}_{+}^{n}$. Such condition number is close in spirit to $C(A)$ but is invariant under row scaling and is defined in terms of a best conditioned solution to (6). This condition number can also be used in the analysis of algorithms (e.g., the analysis in [11] carries over to $\mathscr{C}(A)$ ) and has also been studied as a random variable $[8,12,20]$.

\footnotetext{
${ }^{1}$ Similar remarks hold as well, with natural modifications, for linear conic optimization problems with constrains of the form (3) or (4).
} 
In this paper we show that the definition and key characterization of $\mathscr{C}(A)$ extend to the general multifold conic system (6) for a particular class of norms in $X$. We also provide a maxmin characterization of $C(A)$ which appears to be new, although related to work in [18]. The proof of both facts is, essentially, the same, a feature that emphasizes the close relationship between $C(A)$ and $\mathscr{C}(A)$.

\subsection{Statement of the main result}

Given a triple $(X, K, e)$ with a Banach space $X$, a regular closed convex cone $K \subseteq X$, and a fixed point $e \in \operatorname{int}(K)$, define the "minimum-eigenvalue" function $\lambda_{\min }: X \rightarrow \mathbb{R}$ as

$$
x \mapsto \max \{t \in \mathbb{R}: x-t e \in K\} .
$$

Notice that $\lambda_{\min }$ is positively homogeneous, i.e., it satisfies

$$
\lambda_{\min }(s x)=s \lambda_{\min }(x) \text { for all } s \geq 0 \text { and } x \in X,
$$

and superlinear, i.e., it satisfies

$$
\lambda_{\min }(x+u) \geq \lambda_{\min }(x)+\lambda_{\min }(u) \text { for all } x, u \in X .
$$

Notice also that $x \in K \Leftrightarrow \lambda_{\min }(x) \geq 0$ and $x \in \operatorname{int}(K) \Leftrightarrow \lambda_{\min }(x)>0$.

We shall say that the triple $(X, K, e)$ satisfies the norm compatibility condition if the following condition holds:

$$
\left|\lambda_{\min }(x)-\lambda_{\min }(u)\right| \leq \frac{\|x-u\|}{\|e\|}, \quad \text { for all } x, u \in X .
$$

We will see in Section 2 below that a natural, canonical norm can be associated to any triple $(X, K, e)$ such that the triple satisfies (NC) for this canonical norm. We next proceed with conditioning.

For $j=1, \ldots, r$, let $e_{j} \in \operatorname{int}\left(K_{j}\right)$ be fixed and $\lambda_{\text {min }}^{j}: X_{j} \rightarrow \mathbb{R}$ denote the minimum-eigenvalue function (7) corresponding to the triple $\left(X_{j}, K_{j}, e_{j}\right)$.

Let $\alpha \in \mathbb{R}_{++}^{r}$ be given. Define the condition value of a point $y \in Y \backslash\{0\}$ to be

$$
v_{A, \alpha}(y):=\min _{j=1, \ldots, r} \frac{\lambda_{\min }^{j}\left(A_{j} y\right)}{\alpha_{j}\|y\|} .
$$

Observe that $y$ is a strict solution to (6) if and only if $v_{A, \alpha}(y)>0$. Define the best conditioned value $\bar{v}_{A, \alpha}$ to be

$$
\bar{v}_{A, \alpha}:=\max _{y \neq 0} v_{A, \alpha}(y)
$$


and a best conditioned point to be any point $\bar{y}_{A} \in Y$ that satisfies

$$
v_{A, \alpha}\left(\bar{y}_{A}\right)=\bar{v}_{A, \alpha} .
$$

Notice that $A \in \mathcal{D}$ if and only if $\bar{v}_{A, \alpha}>0, A \in \Sigma$ if and only if $\bar{v}_{A, \alpha}=0$, and $A \in \mathcal{P}$ if and only if $\bar{v}_{A, \alpha}<0$. Notice also that this is valid for all $\alpha \in \mathbb{R}_{++}^{r}$.

We are now ready to state our main results (we delay their proofs to Section 3).

Theorem 1 If each one of the triples $\left(X_{j}, K_{j}, e_{j}\right), j=1, \ldots, r$, satisfies the norm compatibility condition (NC) then

$$
\left|\bar{v}_{A, \alpha}\right|=\min _{\widetilde{A} \in \Sigma} \max _{j=1, \ldots, r} \frac{\left\|A_{j}-\widetilde{A}_{j}\right\|}{\alpha_{j}},
$$

where the norms in the right hand side are the operator norms induced by the norms in $Y$ and $X_{j}$.

It is customary to define condition numbers either as a relativized distance to ill-posedness or as the condition of a best conditioned solution. Theorem 1 shows that both choices lead to the same notion by taking the condition number with respect to $\alpha$ to be

$$
C_{\alpha}(A):=\frac{1}{\left|\bar{v}_{A, \alpha}\right|}
$$

and requiring the norm in $X$ to satisfy $\left\|\left(x_{1}, \ldots, x_{r}\right)\right\|=\max _{j \leq r}\left\|x_{j}\right\|$. Note that the distance to ill-posedness in the right hand side in Theorem 1 is relativized by the vector $\alpha$.

In the previous development we have assumed that $\alpha_{j}>0$ for $j=$ $1, \ldots, r$. From a perturbation theory viewpoint this corresponds to assume that all the $A_{j}$ can be perturbed and that the magnitude of these perturbations are weighted (or relativized) by the $\alpha_{j}$.

We next consider the case where some blocks are rigid, i.e., can not be perturbed. This amounts to setting the corresponding $\alpha_{j}$ to zero. To that end, assume $B \cup N=\{1, \ldots, r\}$ is a partition of $\{1, \ldots, r\}$ with $B \neq \emptyset$. Let $X_{N}=\prod_{j \in N} X_{j}, K_{N}=\prod_{j \in N} K_{j}$, and $A_{N}=\prod_{j \in N} A_{j}$. Write also $\alpha_{N}=\left(\alpha_{j}\right)_{j \in N}$ and $\alpha_{B}=\left(\alpha_{j}\right)_{j \notin N}$. If we only allow perturbations in the blocks $A_{j}$ for $j \in B$, then the following extension of Theorem 1 holds. 
Theorem 2 Assume each one of the triples $\left(X_{j}, K_{j}, e_{j}\right), j=1, \ldots, r$, satisfies the norm compatibility condition. Then, for $A \notin \Sigma$,

$$
\left|\max _{\substack{A_{N} y \in K_{N} \\ y \neq 0}} \min _{j \in B} \frac{\lambda_{\min }^{j}\left(A_{j} y\right)}{\alpha_{j}\|y\|}\right|=\min _{\substack{\widetilde{A} \in \Sigma \\ \widetilde{A}_{N}=A_{N}}} \max _{j \in B} \frac{\left\|A_{j}-\widetilde{A}_{j}\right\|}{\alpha_{j}}
$$

with the convention that the left hand side above is $+\infty$ if

$$
\left\{y: A_{N} y \in K_{N}, y \neq 0\right\}=\emptyset
$$

and the right hand side is $+\infty$ if

$$
\left\{\widetilde{A} \in \Sigma: \widetilde{A}_{N}=A_{N}\right\}=\emptyset .
$$

Furthermore, (9) can be seen as a limit case of Theorem 1. More precisely, for $A \notin \Sigma$,

$$
\left|\max _{A_{N} y \in K_{N}} \min _{j \in B} \frac{\lambda_{\min }^{j}\left(A_{j} y\right)}{\alpha_{j}\|y\|}\right|=\lim _{\substack{\alpha_{B} \text { fixed } \\ \alpha_{N} \downarrow 0}}\left|\bar{v}_{A, \alpha}\right|
$$

and

$$
\min _{\substack{\widetilde{A} \in \Sigma \\ \widetilde{A}_{N}=A_{N}}} \max _{j \in B} \frac{\left\|A_{j}-\widetilde{A}_{j}\right\|}{\alpha_{j}}=\lim _{\substack{\alpha_{B} \text { fixed } \\ \alpha_{N} \downarrow 0}} \min _{\widetilde{A} \in \Sigma} \max _{j \leq r} \frac{\left\|A_{j}-\widetilde{A}_{j}\right\|}{\alpha_{j}} .
$$

Remark 1 The identity (9) in Theorem 2 does not necessarily hold if $A \in$ $\Sigma$. For instance, consider the example $r=2, B=\{1\}, \alpha_{1}=1, X_{1}=\mathbb{R}$, $X_{2}=Y=\mathbb{R}^{2}, K_{1}=\mathbb{R}_{+}, K_{2}=\mathbb{R}_{+}^{2}, A_{1}=\left[\begin{array}{ll}1 & 0\end{array}\right]$, and $A_{2}=\left[\begin{array}{ll}1 & 0 \\ 0 & 0\end{array}\right]$. In this example $A \in \Sigma$ and thus the right hand side in (9) is zero but a simple calculation shows that the left hand side is one.

Nevertheless, when $A \in \Sigma$, a modified version of (9) holds if the set of illposed instances $\Sigma$ is redefined taking into account the relationship between the rigid part $A_{N} Y$ and the cone $K_{N}$.

\section{Examples}

In this section we recall some basic notions, describe various cones, exhibit explicit descriptions of their minimum-eigenvalue functions and canonical norms, and show how Theorems 1 and 2 apply in a number of situations. 


\subsection{Cones and norms}

A pointed cone in $\mathbb{R}^{n}$ is a set $K \subset \mathbb{R}^{n}$ satisfying

(i) for every $x \in \mathbb{R}^{n}$, if $x \in K$ then $\lambda x \in K$ for all $\lambda \geq 0$, and

(ii) $K \cap-K=\{0\}$.

A cone is regular if it is pointed and has non-empty interior. In what follows we assume that all cones are closed, convex and regular.

We first show that the norm compatibility condition for a given triple $(X, K, e)$ can be alternatively stated in terms of the ball

$$
\mathbb{B}:=\{x \in X:\|x\| \leq\|e\|\} .
$$

The equivalent statement will in turn enable us to show that, for any triple $(X, K, e)$, there is a canonical norm on $X$, equivalent to the norm \|\| , for which the norm compatibility condition (NC) holds.

Proposition 1 The norm compatibility condition (NC) holds if and only if

$$
e+\mathbb{B} \subseteq K
$$

Proof. To prove the equivalence between (NC) and (NC') first observe that $(\mathrm{NC})$ can be equivalently phrased as

$$
\left|\lambda_{\min }(x+d)-\lambda_{\min }(x)\right| \leq 1 \quad \text { for all } d \in \mathbb{B} \text { and } x \in X \text {. }
$$

$(\mathrm{NC} ")$

Assume (NC") holds. Then in particular for all $d \in \mathbb{B}$ we have

$$
\lambda_{\min }(e+d)-\lambda_{\min }(e) \geq-1
$$

So

$$
\lambda_{\min }(e+d) \geq-1+\lambda_{\min }(e)=0,
$$

and consequently $e+d \in K$. Since this holds for all $d \in \mathbb{B}$, we get (NC').

Conversely, assume (NC') holds. Let $d \in \mathbb{B}$ and $x \in X$ be given. By the construction of $\lambda_{\min }$ we have

$$
x-\lambda_{\min }(x) e \in K
$$

and by (NC') we have

$$
d+e \in K
$$


Hence $x+d-\left(\lambda_{\min }(x)-1\right) e \in K$. Consequently $\lambda_{\min }(x+d) \geq \lambda_{\min }(x)-1$ by the construction of $\lambda_{\min }$. Thus

$$
\lambda_{\min }(x+d)-\lambda_{\min }(x) \geq-1 .
$$

On the other hand, again by the construction of $\lambda_{\min }$ and by (NC') we have

$$
x+d-\lambda_{\min }(x+d) e \in K
$$

and

$$
-d+e \in K
$$

Hence $x-\left(\lambda_{\min }(x+d)-1\right) e \in K$. Consequently $\lambda_{\min }(x) \geq \lambda_{\min }(x+d)-1$, i.e.,

$$
\lambda_{\min }(x+d)-\lambda_{\min }(x) \leq 1 .
$$

We thus get (NC") from (10) and (11).

Our next statement relies on the gauge function of a set (see, e.g., [4, 29]). Assume $X$ is a real topological vector space and $B \subseteq X$ is such that $0 \in$ $\operatorname{int}(B)$. The gauge function $\gamma_{B}: X \rightarrow \mathbb{R}_{+}$is defined as

$$
\gamma_{B}(x):=\inf \left\{t>0: \frac{1}{t} x \in B\right\}
$$

When $B$ is convex, symmetric (i.e., $B=-B$ ), and bounded, the gauge function $\gamma_{B}$ defines a norm on $X$ (cf. [29, Chapter 2]).

Corollary 1 Assume $(X, K, e)$ is a triple and let $B \subseteq X$ be the largest symmetric set such that $e+B \subseteq K$, that is,

$$
B:=\{x \in X: x+e \in K,-x+e \in K\} .
$$

Then the gauge function $\gamma_{B}$ defines a canonical norm \|\|$_{\mathbf{c}}$ on $X$ for which $(X, K, e)$ satisfies the norm compatibility condition and $\|e\|_{\mathbf{c}}=1$.

Remark 2 (i) The canonical norm of Corollary 1 plays a central role in primal-dual interior-point methods for self-scaled cones. In such context, it is generally denoted as ||$_{e}$. See, e.g., $[22,23,26]$.

(ii) One can prove that a norm satisfies (NC) if and only if the min-width parameter $\tau$ of $K$ defined in [18] satisfies $\tau=1$. In case the norms of some $X_{j}$ do not satisfy (NC), one may extend Theorem 1 to obtain inequalities involving the min-width of the respective cones. 
As some of the examples below illustrate, the canonical norm in Corollary 1 coincides with commonly used norms in a number of cases.

Example 1 (Polyhedral cones) Consider $(X, K, e)=\left(\mathbb{R}^{n}, \mathbb{R}_{+}^{n},(1, \ldots, 1)\right)$. In this case

$$
\lambda_{\min }(x)=\min _{j} x_{j}
$$

and the canonical norm is the infinity norm

$$
\|x\|_{\mathbf{c}}=\|x\|_{\infty}=\max _{j}\left|x_{j}\right|
$$

Example 2 (Second-order cone) Consider $(X, K, e)=\left(\mathbb{R}^{n+1}, \mathcal{Q}^{n},(1,0, \ldots, 0)\right)$ where $\mathcal{Q}^{n}$ is the second-order cone defined to be

$$
\mathcal{Q}^{n}:=\left\{x=\left(x_{0}, \bar{x}\right), \bar{x} \in \mathbb{R}^{n}: x_{0} \geq\|\bar{x}\|_{2}\right\} .
$$

In this case

$$
\lambda_{\min }(x)=\min \left\{x_{0}-\|\bar{x}\|_{2}, x_{0}+\|\bar{x}\|_{2}\right\}=x_{0}-\|\bar{x}\|_{2}
$$

and the canonical norm is the "spectral norm"

$$
\|x\|_{\mathbf{c}}=\max \left\{\left|x_{0}-\|\bar{x}\|_{2}\right|,\left|x_{0}+\|\bar{x}\|_{2}\right|\right\}=\left|x_{0}\right|+\|\bar{x}\|_{2} .
$$

Example 3 (Semidefinite cone) Consider $(X, K, e)=\left(\mathbf{S}^{n}, \mathbf{S}_{+}^{n}, \mathrm{I}\right)$ where $\mathbf{S}^{n}$ is the set of $n \times n$ symmetric matrices and $\mathrm{I}$ is the identity matrix. In this case

$$
\lambda_{\min }(x)=\min _{j=1, \ldots, n} \lambda_{j}(x)
$$

and the canonical norm is the spectral norm

$$
\|x\|_{\mathbf{c}}=\max _{j=1, \ldots, n}\left|\lambda_{j}(x)\right| .
$$

where the $\lambda_{j}(x), j=1, \cdots, n$, are the usual eigenvalues of $x$, i.e., the roots of $p(\lambda):=\operatorname{det}(\lambda \mathrm{I}-x)$,

Example 4 (Cones of positive semidefinite Hermitian matrices) Consider $(X, K, e)$ where $X$ is the real vector $\operatorname{Herm}(n, \mathbb{C})$ space of $n \times n$ Hermitian matrices with complex entries, $K$ is the cone of positive semidefinite Hermitian matrices in $X$, and $e$ is the $n \times n$ identity matrix. In this case

$$
\lambda_{\min }(x)=\min _{j=1, \ldots, n} \lambda_{j}(x)
$$


and the canonical norm is the spectral norm

$$
\|x\|_{\mathbf{c}}=\max _{j=1, \ldots, n}\left|\lambda_{j}(x)\right|
$$

where the $\lambda_{j}(x), j=1, \cdots, n$ are the usual eigenvalues of $x$, i.e., the roots of $p(\lambda):=\operatorname{det}(\lambda \mathrm{I}-x)$ which, it is well known, are real.

\section{Example 5 (Cones of psd Hermitian matrices with quaternions entries)}

Consider $(X, K, e)$ where $X$ is the real vector space $\operatorname{Herm}(n, \mathbb{H})$ of $n \times n$ Hermitian matrices with quaternion entries, $K$ is the cone of positive semidefinite Hermitian matrices in $X$, and $e$ is the $n \times n$ identity matrix. In this case

$$
\lambda_{\min }(x)=\min _{j=1, \ldots, n} \lambda_{j}(x)
$$

and the canonical norm is the spectral norm

$$
\|x\|_{\mathbf{c}}=\max _{j=1, \ldots, n}\left|\lambda_{j}(x)\right| .
$$

where the $\lambda_{j}(x), j=1, \cdots, n$, are the roots (as a univariate polynomial in $\lambda$ ) of the "characteristic polynomial" $\operatorname{det}(\lambda e-x)$ of $X$. This polynomial is defined as follows [16]. Let $J$ be the $2 n \times 2 n$ matrix $\left[\begin{array}{cc}0 & \mathrm{I}_{n} \\ -\mathrm{I}_{n} & 0\end{array}\right]$. Then $\operatorname{Herm}(n, \mathbb{H})$ can be written as $\{z \in \operatorname{Herm}(2 n, \mathbb{C}): \bar{z} J=J z\}$ and, for $z \in$ $\operatorname{Herm}(n, \mathbb{H})$,

$$
\operatorname{det}(z)=\operatorname{Pf}(J z)
$$

where $\operatorname{Pf}(J z)$ is the Pfaffian of $J z$, i.e., the unique polynomial satisfying $\operatorname{Pf}(J)=1, \operatorname{Pf}(J z)^{2}=\operatorname{det}(J z)$. Again, it is well known that the $\lambda_{j}(x)$, $j=1, \ldots, n$, are real. (For a more detailed discussion on Pfaffians, see, e.g., [21].)

Example 6 (Cones of squares in the Albert algebra) Consider $(X, K, e)$ where $X$ is the real vector space of $3 \times 3$ Hermitian matrices with octonion entries $[2,10], K$ is the cone of squares in $X$, i.e., $K=\left\{x^{2}: x \in X\right\}$, and $e$ is the $3 \times 3$ identity matrix. In this case

$$
\lambda_{\min }(x)=\min _{j=1,2,3} \lambda_{j}(x)
$$

and the canonical norm is the "spectral norm"

$$
\|x\|_{\mathbf{c}}=\max _{j=1, \ldots, 3}\left|\lambda_{j}(x)\right|
$$


where $\lambda_{j}(x), j=1,2,3$, are the roots of the "characteristic polynomial"

$$
p(\lambda)=\operatorname{det}(\lambda e-x)=\lambda^{3}-\operatorname{trace}(x) \lambda^{2}+\sigma(x) \lambda-\operatorname{det}(x),
$$

and trace $(x), \sigma(x), \operatorname{det}(x)$ are defined as follows $[13,16]$. For $a, b, c$ octonions and $p, m, n \in \mathbb{R}$,

$$
x=\left[\begin{array}{ccc}
p & a & \bar{b} \\
\bar{a} & m & c \\
b & \bar{c} & n
\end{array}\right]
$$

$$
\begin{aligned}
\operatorname{trace}(x) & =p+m+n \\
\sigma(x) & =p m+m n+p n-|a|^{2}-|b|^{2}-|c|^{2} \\
\operatorname{det}(x) & =p m n+b(a c)+\overline{b(a c)}-n|a|^{2}-m|b|^{2}-p|c|^{2}
\end{aligned}
$$

Note, we write $b(a c)$ to emphasize the order of the multiplications in the non-associative ring of octonions. Just as in the previous examples, $\lambda_{1}, \lambda_{2}$, and $\lambda_{3}$ are real.

Example 7 (Cone of squares in Euclidean Jordan Algebras) Consider $(X, K, e)=(\mathcal{E}, \mathcal{K}, e)$ where $\mathcal{E}$ is an Euclidean Jordan algebra, $\mathcal{K}$ is the closure of the cone of squares in $\mathcal{E}$ and $e \in \mathcal{K}$ is the identity element [15]. In this case

$$
\lambda_{\min }(x)=\min _{j=1, \ldots, r} \lambda_{j}(x)
$$

and the canonical norm is the spectral norm

$$
\|x\|_{\mathbf{c}}=\max _{j=1, \ldots, r}\left|\lambda_{j}(x)\right|
$$

where the $\lambda_{j}(x), j=1, \ldots, r$, are the Jordan algebra eigenvalues of $x$, i.e., the eigenvalues of the characteristic polynomial $\operatorname{det}(\lambda e-x)$ for a suitable homogeneous polynomial det, [15, Chapter 3]. Notice that this case encompasses Examples 1, 2, 3, 4, 5, and 6 above. We will touch on this theme again in Theorem 3 below.

Example 8 (Hyperbolicity cones) Let $e \in \mathbb{R}^{n}$ and $p \in \mathbb{R}\left[X_{1}, \ldots, X_{n}\right]$ be a complete hyperbolic polynomial in the direction $e$, i.e., a homogeneous polynomial satisfying that, for all $x \in \mathbb{R}^{n}$, the univariate polynomial $\lambda \mapsto$ $p(\lambda e-x)$ has only real roots, and at least one of them is non-zero for $x \neq$ 0. (For a detailed exposition on hyperbolic polynomials, see, e.g., [3, 27].) Consider $(X, K, e)=\left(\mathbb{R}^{n}, C(p, e), e\right)$ where $C(p, e)$ is the closure of the 
hyperbolicity cone for $p$ in the direction $e$, i.e., $C(p, e)$ is the closure of the connected component of the set $\{x: p(x)>0\}$ that contains $e$. In this case

$$
\lambda_{\min }(x)=\min _{j=1, \ldots, d} \lambda_{j}(x)
$$

where $\lambda_{j}(x), j=1, \ldots, d=\operatorname{deg}(p)$, are the roots of the polynomial $\lambda \mapsto$ $p(\lambda e-x)$, and

$$
\|x\|_{\mathbf{c}}=\max _{j=1, \ldots, d}\left|\lambda_{j}(x)\right|
$$

Example 9 (Non-negative, finitely spanned, functions on a compact domain) Assume $d \in \mathbb{N}, D \subseteq \mathbb{R}^{n}$ is a nonempty compact set, and $f_{0}, \ldots, f_{d}$ are continuous, real-valued functions defined on $D$, with $f_{0}(x)=1$, for all $x \in D$. Consider the triple $(X, K, e)$ where

$$
\begin{gathered}
X=\operatorname{span}\left\{f_{0}, \ldots, f_{d}\right\}, \\
K=\{f \in X: f(x) \geq 0 \text { for all } x \in D\},
\end{gathered}
$$

and $e \in X$ is the constant function $f_{0}$. In this case, for $f \in X$,

$$
\lambda_{\min }(f)=\min _{x \in D} f(x)
$$

and

$$
\|f\|_{\mathbf{c}}=\max _{x \in D}|f(x)|
$$

\subsection{Cone Reducibility}

We briefly recall the concepts of dual space and dual cone (for details see $[28]$ ). The dual of a real Banach space $X$ is the Banach space $X^{*}=L(X, \mathbb{R})$, i.e., the space of bounded real-valued linear mappings defined on $X$. Given $x \in X$ and $u \in X^{*}$ it is customary to write $\langle u, x\rangle$ for $u(x)$. The norm \|\| in $X$ induces a dual norm \|\|$^{*}$ in $X^{*}$. More precisely, for $u \in X^{*}$,

$$
\|u\|^{*}=\max \{|\langle u, x\rangle|: x \in X,\|x\| \leq 1\} .
$$

The dual of a cone $K \subseteq X$ is the cone

$$
K^{*}=\left\{u \in X^{*}:\langle u, x\rangle \geq 0, \text { for all } x \in K\right\} .
$$

If $X$ has an inner product $(\mid)$ then $X^{*}$ can be identified with $X$ by mapping $u \in X^{*}$ to the unique element $v \in X$ such that $\langle u, x\rangle=(v \mid x)$ for all $x \in X$. In such case we say that $K$ is self-dual if $K^{*}=K$. We say that a cone $K$ is 
homogeneous if for all $x, u \in \operatorname{int}(K)$ there exists $g \in \operatorname{Aut}(\operatorname{int}(K))$ such that $g x=u$, where Aut $(\operatorname{int}(K))=\{g \in \mathrm{GL}(X): g(\operatorname{int}(K))=\operatorname{int}(K)\}$. A cone is symmetric if it is self-dual and homogeneous [15].

Symmetric cones coincide with self-scaled cones, a class of cones that plays a central role in interior-point methods [22]. Nesterov and Todd identified the properties of self-scaled cones as the fundamental building blocks for the development of symmetric primal-dual interior-point algorithms [23, 26] for conic programs over these cones. Symmetric cones have been extensively studied in other areas of mathematics. They satisfy a unique factorization property which we state in the following result.

Theorem 3 ([15]) A cone is symmetric if and only if it is isomorphic to the cone of squares of an Euclidean Jordan algebra. Furthermore, any symmetric cone decomposes in a unique way (up to ordering) as a product

$$
K=K_{1} \times \ldots \times K_{r}
$$

where the $K_{i}$ belong to one of the following five classes of cones:

(i) the class of second-order cones,

(ii) the class of cones of symmetric positive semidefinite matrices,

(iii) the class of cones of Hermitian positive semidefinite matrices with complex entries,

(iv) the class of cones of Hermitian positive semidefinite matrices with quaternion entries, and

(v) the cone of squares in the Albert algebra.

Because second-order conic feasibility over a single second-order cone can be solved in closed form, all interesting examples of second-order conic feasiblity problems are written in terms of a non-trivial product of secondorder cones as in Theorem 3 (see, e.g., $[1,30]$ ).

Given $X$ and $K$ one may wonder how the canonical norm and the minimum-eigenvalue constructs depend on different factorizations of $(X, K)$. The following two propositions settle this question.

Proposition 2 Let $X=X_{1} \times \ldots \times X_{r}, K=K_{1} \times \ldots \times K_{r}$, and $e=$ $\left(e_{1}, \ldots, e_{r}\right)$ with $e_{j} \in \operatorname{int}\left(K_{j}\right)$. Then, for all $x=\left(x_{1}, \ldots, x_{r}\right) \in X$,

$$
\lambda_{\min }(x)=\min \left\{\lambda_{\min }^{1}\left(x_{1}\right), \ldots, \lambda_{\min }^{r}\left(x_{r}\right)\right\} .
$$


where $\lambda_{\min }^{j}$ is the minimum eigenvalue associated to $\left(X_{j}, K_{j}, e_{j}\right)$ and $\lambda_{\min }$ that associated to $(X, K, e)$.

Proof. From (7) it follows that, for $x=\left(x_{1}, \ldots, x_{r}\right) \in X$,

$$
\begin{aligned}
\lambda_{\min }(x) & =\max \{t \in \mathbb{R}: x-t e \in K\} \\
& =\max \left\{t \in \mathbb{R}: x_{j}-t e_{j} \in K_{j}, \text { for } j=1, \ldots, r\right\} \\
& =\min _{j=1, \ldots, r} \max \left\{t \in \mathbb{R}: x_{j}-t e_{j} \in K_{j}\right\} \\
& =\min \left\{\lambda_{\min }^{1}\left(x_{1}\right), \ldots, \lambda_{\min }^{r}\left(x_{r}\right)\right\} .
\end{aligned}
$$

Proposition 3 Let $X=X_{1} \times \ldots \times X_{r}, K=K_{1} \times \ldots \times K_{r}$, and $e=$ $\left(e_{1}, \ldots, e_{r}\right)$ with $e_{j} \in \operatorname{int}\left(K_{j}\right)$. Then, for all $x=\left(x_{1}, \ldots, x_{r}\right) \in X$,

$$
\|x\|_{\mathbf{c}}=\max _{j=1, \ldots, r}\left\|x_{j}\right\|_{\mathbf{c}, j}
$$

where \|\|$_{\mathbf{c}, j}$ is the canonical norm associated to $\left(X_{j}, K_{j}, e_{j}\right)$ and \|\|$_{\mathbf{c}}$ that associated to $(X, K, e)$. In particular, the restriction of \|\|$_{\mathbf{c}}$ to $X_{j}$ is \|\|$_{\mathbf{c}, j}$.

Proof. First, we claim that

$$
B=B_{1} \times \cdots \times B_{r} .
$$

Indeed, given $d=\left(d_{1}, \ldots, d_{r}\right) \in X$,

$$
\begin{aligned}
d \in B & \Leftrightarrow e+d, e-d \in K \\
& \Leftrightarrow e_{j}+d_{j}, e_{j}-d_{j} \in K_{j}, \text { for } j=1, \ldots, r \\
& \Leftrightarrow d_{j} \in B_{j}, \text { for } j=1, \ldots, r \\
& \Leftrightarrow d \in B_{1} \times \cdots \times B_{r} .
\end{aligned}
$$

From (12) it follows that, for $x=\left(x_{1}, \ldots, x_{r}\right) \in X$,

$$
\begin{aligned}
\|x\|_{\mathbf{c}} & =\inf \left\{t: \frac{1}{t} x \in B\right\} \\
& =\inf \left\{t: \frac{1}{t} x_{j} \in B_{j} \text { for } j=1, \ldots, r\right\} \\
& =\max _{j=1, \ldots, r} \inf \left\{t: \frac{1}{t} x_{j} \in B_{j}\right\} \\
& =\max _{j=1, \ldots, r}\left\|x_{j}\right\|_{\mathbf{c}, j} .
\end{aligned}
$$


We have already mentioned that we endow $L(Y, X)$ with the operator norm with respect to the norms in $Y$ and $X$. Therefore, the canonical norm in $X$ induces a canonical norm in $L(Y, X)$. In particular, in case $X=X_{1} \times \ldots \times X_{r}$, Proposition 3 yields the relation

$$
\|A\|=\max _{j=1, \ldots, r}\left\|A_{j}\right\|
$$

for the canonical norms in $L(Y, X)$ and those in $L\left(Y, X_{j}\right)$.

Remark 3 Note that the factorization in Theorem 3, together with Proposition 3 and Examples 2 to 6, yield expressions for the canonical norm for every symmetric cone. If the factorization is explicit, then the expressions for the canonical norm are explicit as well.

\subsection{Condition numbers and the choice of $\alpha$}

We mentioned in $§ 1.1$ the role of Renegar's condition number in the analysis of iterative algorithms for conic feasibility problems. We also mentioned there that, in the case of polyhedral cones, the condition number $\mathscr{C}(A)$ exploited the reducibility of the cone $\mathbb{R}_{+}^{n}$. This condition number was extended in [9] to the case of second-order conic systems (Example 2 above). We next show how these condition numbers are obtained by appropriately selecting $\alpha$.

Assume a factorization $X=X_{1} \times \ldots \times X_{r}$ and $K=K_{1} \times \ldots \times K_{r}$. Basic choices for $\alpha$ are:

(1) $\alpha_{j}=\|A\|$ for $j=1, \ldots, r$.

(2) $\alpha_{j}=\left\|A_{j}\right\|$ for $j=1, \ldots, r$.

The first choice leads to Renegar's condition number $C(A)$ for the norm in $L(Y, X)$ defined by

$$
\|A\|=\max _{j=1, \ldots, r}\left\|A_{j}\right\|
$$

because in this case

$$
C_{\alpha}(A)=\frac{\|A\|}{\min _{\widetilde{A} \in \Sigma} \max _{j=1, \ldots, r}\left\|A_{j}-\widetilde{A}_{j}\right\|}=\frac{\|A\|}{\min _{\widetilde{A} \in \Sigma}\|A-\widetilde{A}\|}=C(A) .
$$

Theorem 1 takes then the form of a minmax characterization of the distance to ill-posedness (and therefore, of $C(A)$ ). This is related to work in [18]. 
A natural question is whether different factorizations of $(X, K)$ may lead to different values of $\|A\|$ in (13) above and, thus, to different values of $C(A)$. This is not the case for "compatible" factorizations and the canonical norms.

Lemma 1 Let $(X, K, e)$ be a triple. Let also $X_{1} \times \ldots \times X_{r}$ and $X_{1}^{\prime} \times \ldots \times X_{q}^{\prime}$ be different factorizations of $X$ and $K_{1} \times \ldots \times K_{r}$ and $K_{1}^{\prime} \times \ldots \times K_{q}^{\prime}$ the corresponding factorizations of $K$. Define $e_{j}=\pi_{j}(e)$ for $j=1, \ldots, r$ and $e_{i}^{\prime}=\pi_{i}^{\prime}(e)$ for $i=1, \ldots, q$, where $\pi_{j}$ and $\pi_{i}^{\prime}$ are the projections of $X$ over $X_{j}$ and $X_{i}^{\prime}$, respectively. Then, for all $A \in L(Y, X)$,

$$
\max _{j=1, \ldots, r}\left\|A_{j}\right\|_{\mathbf{c}, j}=\max _{i=1, \ldots, q}\left\|A_{i}^{\prime}\right\|_{\mathbf{c}, i}
$$

where $A_{j}$ and $A_{i}^{\prime}$ are the projections of $A$ over $X_{j}$ and $X_{i}^{\prime}$, respectively. In particular, both factorizations yield the same $C(A)$.

Proof. $\quad$ It is an immediate consequence of Proposition 3.

The second choice of $\alpha$ above leads to (extensions of) the condition number $\mathscr{C}(A)$ introduced in [7].

The discusion above assumes $\alpha_{j}>0$ for all $j=1, \ldots, r$. If some cones are rigid, say $K=K_{1} \times \ldots \times K_{r} \times K_{N}$ with $\alpha_{j}>0$ as above and $\alpha_{N}=0$, then, letting $B=\{1, \ldots, r\}$, one defines

$$
C(A)=\frac{\left\|A_{B}\right\|}{\min _{\substack{\widetilde{A} \in \Sigma \\ \widetilde{A}_{N}=A_{N}}} \max _{j \in B}\left\|A_{j}-\widetilde{A}_{j}\right\|}
$$

and

$$
\mathscr{C}(A)=\frac{1}{\left|\max _{\substack{A_{N} y \in K_{N} \\ y \neq 0}} \min _{j \in B} \frac{\lambda_{\min }^{j}\left(A_{j} y\right)}{\left\|A_{j}\right\|\|y\|}\right|} .
$$

The proof of the following proposition is an immediate consequence of the fact that $\left\|A_{j}\right\| \leq\|A\|$ for all $j=1, \ldots, r$.

Proposition 4 For all $A \in L(Y, X), \mathscr{C}(A) \leq C(A)$. This holds as well if some factors of $A$ are rigid.

We next see how the choices of $\alpha$ above materialize in the case of polyhedral conic systems. 
Example 1 (revisited) Recall, $X=\mathbb{R}^{n}, Y=\mathbb{R}^{m}$, and $K=\mathbb{R}_{+}^{n}$.

(i) Consider the case $r=1$. We do not decompose $K$. In this case we take $e=(1, \ldots, 1)$ and, we have seen, $\|x\|=\|x\|_{\infty}$. This induces the canonical norm in $L(Y, X)$ given by

$$
\|A\|=\|A\|_{Y \infty}=\max _{\|y\|=1}\|A y\|_{\infty}=\max _{\|y\|=1} \max _{j=1, \ldots, n}\left|A_{j} y\right| .
$$

We now take $\alpha=\|A\|$. Theorem 1 , and the fact that $\lambda_{\min }(x)=\min _{j \leq n} x_{j}$, give then the following characterization of Renegar's condition number

$$
C(A)=\frac{\|A\|}{\operatorname{dist}(A, \Sigma)}=\frac{\|A\|}{\left|\max _{\|y\|=1} \min _{j=1, \ldots, n} A y\right|} .
$$

(ii) Consider now the case $r=n$. Here we take $K_{j}=[0,+\infty)$ and $e_{j}=1$, for $j=1, \ldots, r$. We obtain the canonical norm $\|x\|=|x|$ and the minimumeigenvalue $\lambda_{\min }(x)=x$. The former induces the canonical norm in $L(Y, X)$ given by

$$
\|A\|=\max _{j=1, \ldots, n}\left\|A_{j}\right\|=\max _{j=1, \ldots, n\|y\|=1} \max _{\| y}\left|A_{j} y\right|
$$

that is, as in case (i) above. Again, take $\alpha_{j}=\|A\|$ for all $j$. Then, not surprisingly, Theorem 1 characterizes $C(A)$ by (14) as well.

(iii) We now take $r, K_{j}$ and $e_{j}$ as in (ii) but choose instead $\alpha_{j}=\left\|A_{j}\right\|=$ $\max _{\|y\|=1}\left|A_{j} y\right|$. In this case Theorem 1 gives us the well-known [7] characterization of $\mathscr{C}(A)$,

$$
\mathscr{C}(A):=\frac{1}{\left|\bar{v}_{A, \alpha}\right|}=\frac{1}{\min _{\widetilde{A} \in \Sigma} \max _{j=1, \ldots, n} \frac{\left\|A_{j}-\widetilde{A}_{j}\right\|}{\left\|A_{j}\right\|}} .
$$

(iv) For $M \in \mathbb{R}^{n \times m}$ consider the system

$$
\begin{array}{r}
M y \geq 0 \\
y \geq 0 .
\end{array}
$$

This system can be thought of as a special case of the above with $A=(M, \mathrm{I})$. The identity matrix I, however, should be considered to be rigid (not subject to perturbations) and its corresponding $\alpha_{\mathrm{I}}$ be then set to 0 . 
Taking $r=2$ (two bloks, corresponding to $M$ and I), $\alpha_{M}=\|M\|=$ $\max _{j=1, \ldots, n} \max _{\|y\|=1}\left|M_{j} y\right|$ we obtain (in the right hand side of (9)) Renegar's condition number and Theorem 2 shows that

$$
C(M):=\frac{\|M\|}{\min _{\widetilde{M} \in \Sigma}\|M-\widetilde{M}\|}=\frac{\|M\|}{\min _{\widetilde{M} \in \Sigma} \max _{j \leq n}\left\|M_{j}-\widetilde{M}_{j}\right\|}=\frac{\|M\|}{\left|\max _{y \geq 0} \min _{j \leq n} \frac{M_{j} y}{\|y\|}\right|}
$$

Finally, taking $r=n$ and $\alpha_{j}=\left\|M_{j}\right\|=\max _{\|y\|=1}\left|M_{j} y\right|, j=1, \ldots, n$, we obtain $\mathscr{C}(M)$ in the left hand side of (9) and now Theorem 2 shows that

$$
\mathscr{C}(M):=\frac{1}{\left|\max _{y \geq 0} \min _{j \leq n} \frac{M_{j} y}{\left\|M_{j}|\||\|| \mid\right.}\right|}=\frac{1}{\min _{\widetilde{M} \in \Sigma} \max _{j \leq n} \frac{\left\|M_{j}-\widetilde{M}_{j}\right\|}{\left\|M_{j}\right\|}} .
$$

We have revisited Example 1 to see how Theorems 1 and 2, together with appropriate choices of $\alpha$, yield characterizations of $C(A)$ and $\mathscr{C}(A)$ in the case of polyhedral conic systems, possibly with rigid components. The other examples in $\S 2.1$, and arbitrary products of them, may be similarly dealt with. We will not do so to avoid being repetitious.

\section{Proof of the main results}

We present the proof of Theorem 1 for the special case $\alpha=(1, \ldots, 1)$. The proof for general $\alpha \in \mathbb{R}_{++}^{r}$ is a straightforward modification. To simplify notation, we will drop $\alpha$ from all expressions. Also, note that the result is trivial when $\bar{v}_{A}=0$. Therefore, we will assume that $\bar{v}_{A} \neq 0$. For ease of exposition, we split the proof of Theorem 1 into two parts, namely Propositions 5 and 6 .

\section{Proposition 5}

$$
\left|\bar{v}_{A}\right| \leq \min _{\widetilde{A} \in \Sigma} \max _{j=1, \ldots, r}\left\|A_{j}-\widetilde{A}_{j}\right\| .
$$

Proof. Assume $\widetilde{A}$ is such that

$$
\max _{j=1, \ldots, r}\left\|A_{j}-\widetilde{A}_{j}\right\|<\left|\bar{v}_{A}\right| .
$$

We need to prove that $\widetilde{A} \notin \Sigma$ i.e., $\widetilde{A} \in \mathcal{P} \cup \mathcal{D}$. 
Let $\bar{y}_{A} \in Y$ be such that $v_{A}\left(\bar{y}_{A}\right)=\bar{v}_{A}$. Assume without loss of generality $\left\|\bar{y}_{A}\right\|=1$. Because each $\left(X_{j}, K_{j}, e_{j}\right)$ satisfies the norm compatibility condition it follows that, for all $\widetilde{A}$ and $y \in Y \backslash\{0\}$,

$$
\begin{aligned}
\left|\lambda_{\text {min }}^{j}\left(A_{j} y\right)-\lambda_{\min }^{j}\left(\widetilde{A}_{j} y\right)\right| & \leq\left\|A_{j}-\widetilde{A}_{j}\right\|\|y\| \\
& \leq \max _{j=1, \ldots, r}\left\|A_{j}-\widetilde{A}_{j}\right\|\|y\| \\
& <\left|\bar{v}_{A}\right|\|y\| .
\end{aligned}
$$

In particular

$$
\left|\lambda_{\min }^{j}\left(A_{j} \bar{y}_{A}\right)-\lambda_{\min }^{j}\left(\widetilde{A}_{j} \bar{y}_{A}\right)\right|<\left|\bar{v}_{A}\right| .
$$

We now consider the cases $\bar{v}_{A}<0$ and $\bar{v}_{A}>0$ separately.

Case 1: $\bar{v}_{A}>0$. In this case $A \in \mathcal{D}$. From (8), (16) and the equality $v_{A}\left(\bar{y}_{A}\right)=\bar{v}_{A}$, we get, for $j=1, \ldots, r$,

$$
\begin{aligned}
\lambda_{\text {min }}^{j}\left(\widetilde{A}_{j} \bar{y}_{A}\right) & \geq \lambda_{\text {min }}^{j}\left(A_{j} \bar{y}_{A}\right)-\left|\lambda_{\min }^{j}\left(A_{j} \bar{y}_{A}\right)-\lambda_{\min }^{j}\left(\widetilde{A}_{j} \bar{y}_{A}\right)\right| \\
& >\bar{v}_{A}-\bar{v}_{A}=0 .
\end{aligned}
$$

Therefore

$$
v_{\widetilde{A}}\left(\bar{y}_{A}\right)=\min _{j \leq r} \lambda_{\min }^{j}\left(\widetilde{A}_{j} \bar{y}_{A}\right)>0
$$

which shows that $\bar{y}_{A}$ is a strict solution for $\widetilde{A}$ and consequently, that $\widetilde{A} \in \mathcal{D}$.

Case 2: $\bar{v}_{A}<0$. In this case $A \in \mathcal{P}$. Let $y \in Y \backslash\{0\}$ be given. Since $\bar{v}_{A}<0$, we must have $v_{A}(y)<0$. Let $\bar{j}$ be such that $\frac{\lambda_{\min }^{\bar{j}}\left(A_{\bar{j}} y\right)}{\|y\|}=v_{A}(y)$. We claim that $\frac{\lambda_{\min }^{\bar{j}}\left(\widetilde{A}_{\bar{j}} y\right)}{\|y\|}<0$. Indeed, assume it is not. Then, using (15),

$$
\begin{aligned}
\frac{\lambda_{\min }^{j}\left(\widetilde{A}_{j} y\right)}{\|y\|}-v_{A}(y) & =\frac{\lambda_{\min }^{j}\left(\widetilde{A}_{j} y\right)}{\|y\|}-\frac{\lambda_{\min }^{j}\left(A_{j} y\right)}{\|y\|} \\
& =\left|\frac{\lambda_{\min }^{j}\left(A_{j} y\right)}{\|y\|}-\frac{\lambda_{\min }^{j}\left(\widetilde{A}_{j} y\right)}{\|y\|}\right| \\
& <-\bar{v}_{A} \\
& \leq-v_{A}(y) .
\end{aligned}
$$

It follows that $\frac{\lambda_{\min }^{j}\left(\widetilde{A}_{j} y\right)}{\|y\|}<0$, a contradiction.

We thus have

$$
\bar{v}_{\widetilde{A}}=\max _{y \neq 0} v_{\widetilde{A}}(y)=\max _{y \neq 0} \min _{j \leq r} \frac{\lambda_{\min }^{j}\left(\widetilde{A}_{j} y\right)}{\|y\|}<0
$$


that is, $\widetilde{A} \in \mathcal{P}$.

Recall, given Banach spaces $X$ and $Y$ and a linear mapping $A \in L(Y, X)$ its adjoint $A^{*} \in L\left(X^{*}, Y^{*}\right)$ is the unique linear mapping that satisfies

$$
\langle v, A y\rangle=\left\langle A^{*} v, y\right\rangle, \quad \text { for all } v \in X^{*}, y \in Y .
$$

\section{Proposition 6}

$$
\left|\bar{v}_{A}\right| \geq \min _{\widetilde{A} \in \Sigma} \max _{j=1, \ldots, r}\left\|A_{j}-\widetilde{A}_{j}\right\| .
$$

Proof. We consider the cases $\bar{v}_{A}<0$ and $\bar{v}_{A}>0$ separately.

Case 1: $\bar{v}_{A}<0$. In this case $A \notin \mathcal{D}$ so it suffices to show that for all $\delta>0$ there exists $\widetilde{A} \in \mathcal{D}$ such that, for all $j=1, \ldots, r,\left\|A_{j}-\widetilde{A}_{j}\right\| \leq\left|\bar{v}_{A}\right|+\delta$. Let $\bar{y}_{A} \in Y$ be such that $v_{A}\left(\bar{y}_{A}\right)=\bar{v}_{A}$. Assume without loss of generality $\left\|\bar{y}_{A}\right\|=1$. By the Hahn-Banach Theorem [28, Theorem 5.20], there exists $v \in Y^{*}$ such that $\left\langle v, \bar{y}_{A}\right\rangle=\left\|\bar{y}_{A}\right\|=1$ and $\|v\|^{*}=1$. For $j=1, \ldots, r$, consider $\widetilde{A} \in L\left(Y, X_{j}\right)$ given by

$$
\widetilde{A}_{j}=A_{j}-\left(\bar{v}_{A}-\delta\right)\langle v, \cdot\rangle e_{j} .
$$

We claim that $\widetilde{A} \in \mathcal{D}$. To see this, first notice that $A_{j} \bar{y}_{A}-\bar{v}_{A} e_{j} \in K_{j}$ because $\bar{v}_{A}=v_{A}\left(\bar{y}_{A}\right) \leq \lambda_{\min }^{j}\left(A_{j} \bar{y}_{A}\right)=\max \left\{t \mid A_{j} \bar{y}_{A}-t e_{j} \in K_{j}\right\}$. Therefore,

$$
\widetilde{A}_{j} \bar{y}_{A}=A_{j} \bar{y}_{A}-\left(\bar{v}_{A}-\delta\right)\left\langle v, \bar{y}_{A}\right\rangle e_{j}=\left(A_{j} \bar{y}_{A}-\bar{v}_{A} e_{j}\right)+\delta e_{j} \in \operatorname{int}\left(K_{j}\right)
$$

since $K_{j}$ is convex and $e_{j} \in \operatorname{int}\left(K_{j}\right)$. This show that $\bar{y}_{A}$ is a strict solution for $\widetilde{A}$. To finish, observe that $\left\|A_{j}-\widetilde{A}_{j}\right\|=\left\|\left(\bar{v}_{A}-\delta\right)\langle v, \cdot\rangle e_{j}\right\| \leq\left|\bar{v}_{A}-\delta\right|\|v\|^{*}\left\|e_{j}\right\|=$ $\left|\bar{v}_{A}\right|+\delta$.

Case 2: $\bar{v}_{A}>0$. In this case $A \in \mathcal{D}$ so it suffices to show that there exists $\widetilde{A} \notin \mathcal{D}$ such that, for all $j=1, \ldots, r,\left\|A_{j}-\widetilde{A}_{j}\right\| \leq \bar{v}_{A}$. Let $e=\left(e_{1}, \ldots, e_{r}\right) \in$ $K=K_{1} \times \ldots \times K_{r}$. From Lemma 2 it follows that for $y \in Y \backslash\{0\}$

$$
\begin{aligned}
v_{A}(y) & =\min _{j \leq r} \frac{\lambda_{\min }^{j}\left(A_{j} y\right)}{\|y\|} \\
& =\frac{1}{\|y\|} \lambda_{\min }(A y) \\
& =\frac{1}{\|y\|} \max \{t: A y-t e \in K\} .
\end{aligned}
$$

Then, taking maxima on both sides above,

$$
\bar{v}_{A}=\max _{y \neq 0} v_{A}(y)=\max _{\|y\|=1} \max \{t: A y-t e \in K\} .
$$


Since $\bar{v}_{A}>0$ we may rewrite the above as a maximum over a convex set

$$
\bar{v}_{A}=\max _{\substack{A y-t e \in K \\\|y\| \leq 1}} t
$$

Consider the Lagrangian dual (see [4]) of (17),

$$
\begin{aligned}
& \min _{x \in K^{*}} \max _{\substack{\|y\| \leq 1 \\
t \in \mathbb{R}}} t+\langle x, A y-t e\rangle=\min _{x \in K^{*}} \max _{\substack{\|y\| \leq 1 \\
t \in \mathbb{R}}} t(1-\langle x, e\rangle)+\langle x, A y\rangle \\
& =\min _{\substack{x \in K^{*} \\
\langle x, e\rangle=1}} \max _{\|y\| \leq 1}\left\langle A^{*} x, y\right\rangle \\
& =\min _{\substack{x \in K^{*} \\
\langle x, e\rangle=1}}\left\|A^{*} x\right\|^{*} .
\end{aligned}
$$

Since both (17) and (18) are convex programs and satisfy the Slater condition, by $\left[4\right.$, Theorem 4.3.7], they attain the same optimal value $\bar{v}_{A}$. Hence there exists $\bar{x} \in K^{*}$ such that $\left\|A^{*} \bar{x}\right\|^{*}=\bar{v}_{A}$ and $\langle\bar{x}, e\rangle=1$. Let $\widetilde{A}_{j}=$ $A_{j}-\left\langle A_{j}^{*} \bar{x}, \cdot\right\rangle e_{j}$. We claim that $\widetilde{A} \notin \mathcal{D}$ for otherwise there would be $y \in Y$ and $\epsilon>0$ such that $\widetilde{A} y-\epsilon e \in K$ and, therefore,

$$
\begin{aligned}
0 & \leq\langle\bar{x}, \widetilde{A} y-\epsilon e\rangle \quad \text { because } \bar{x} \in K^{*} \\
& =\left\langle\bar{x}, A y-\left(\left\langle A^{*} \bar{x}, y\right\rangle+\epsilon\right) e\right\rangle \\
& =-\epsilon \quad \text { because }\langle\bar{x}, e\rangle=1 \\
& <0,
\end{aligned}
$$

which is a contradiction. Hence $\widetilde{A} \notin \mathcal{D}$. To finish, observe that

$$
\left\|\widetilde{A}_{j}-A_{j}\right\|=\left\|\left\langle A_{j}^{*} \bar{x}, \cdot\right\rangle e_{j}\right\|=\left\|A_{j}^{*} \bar{x}\right\|^{*}=\bar{v}_{A} .
$$

We next prove Theorem 2. We will need the following result.

Lemma 2 Assume $A \notin \Sigma$. If $\exists y \neq 0\left(A_{N} y \in \partial K_{N} \& A_{B} y \in \operatorname{int}\left(K_{B}\right)\right)$ then $\exists y^{\prime} \neq 0\left(A_{N} y^{\prime} \in \operatorname{int}\left(K_{N}\right) \& A_{B} y^{\prime} \in \operatorname{int}\left(K_{B}\right)\right)$.

Proof. Denote $\mathbf{1}=(1,1, \ldots, 1) \in \mathbb{R}^{d}$. By hypothesis, $v_{A, \mathbf{1}}(y)=0$ and so $\bar{v}_{A, \mathbf{1}} \geq 0$. Since $A \notin \Sigma, \bar{v}_{A, \mathbf{1}}>0$ and so, there is $y^{\prime} \neq 0$ such that $v_{A, \mathbf{1}}\left(y^{\prime}\right)>0$. But this implies $A_{N} y^{\prime} \in \operatorname{int}\left(K_{N}\right)$ and $\left.A_{B} y^{\prime} \in \operatorname{int}\left(K_{B}\right)\right)$. 
Proof of TheOrem 2. We first show that

$$
\left\{y \neq 0 \mid A_{N} y \in K_{N}\right\}=\emptyset \Longleftrightarrow\left\{\widetilde{A} \in \Sigma \mid \widetilde{A}_{N}=A_{N}\right\}=\emptyset .
$$

This will show that the left hand side in (9) is $+\infty$ if and only if so is the right hand side.

For the only if direction, assume there exists $\widetilde{A}_{B}$ such that $\mathscr{A}=\left(\widetilde{A}_{B}, A_{N}\right) \in$ $\Sigma$. The latter implies that $\bar{v}_{\mathscr{A}, \mathbf{1}}=0$. Hence, there exists $\bar{y} \in S_{Y}:=\{y \in$ $Y \mid\|y\|=1\}$ such that $\min _{j \leq r} \lambda_{\text {min }}^{j}\left(\mathscr{A}_{j} \bar{y}\right)=0$ and, therefore, such that $\lambda_{\text {min }}^{j}\left(\mathscr{A}_{j} \bar{y}\right) \geq 0$ for $j=1, \ldots, r$. But this implies that $A_{N} \bar{y} \in K_{N}$.

For the if direction, assume there exists $\bar{y} \neq 0$ such that $A_{N} \bar{y} \in K_{N}$. Let $\mathcal{A}=\left(0, A_{N}\right)$. Then, for all $y \neq 0$, and since $0 y=0 \in \partial K_{B}$,

$$
v_{\mathcal{A}, \mathbf{1}}(y)=\min _{j \leq r} \lambda_{\min }^{j}\left(\mathcal{A}_{j} y\right) \leq 0 .
$$

This implies that $\bar{v}_{\mathcal{A}, \mathbf{1}} \leq 0$. But $v_{\mathcal{A}, \mathbf{1}}(\bar{y})=0$ since $A_{N} \bar{y} \in K_{N}$. Therefore $\bar{v}_{\mathcal{A}, \mathbf{1}}=0$ which implies $\mathcal{A} \in \Sigma$.

We now assume that the sets above are non-empty and take limits when $\alpha_{N} \rightarrow 0$. We will show that both the left and right hand sides of the equality in Theorem 1 tend, when $\alpha_{N} \rightarrow 0$, to the corresponding sides in (9). This equation will hold then since Theorem 1 does.

Recall, the left hand side in the equality of Theorem 1 is

$$
\left|\max _{y \in S_{Y}} \min _{j \leq r} \frac{\lambda_{\min }^{j}\left(A_{j} y\right)}{\alpha_{j}}\right| .
$$

Take $y \in S_{Y}$ such that $A_{N} y \in \operatorname{int}\left(K_{N}\right)$. Then

$$
\begin{gathered}
\forall j \in N \frac{\lambda_{\min }^{j}\left(A_{j} y\right)}{\alpha_{j}}>0 \\
\Longrightarrow \quad \forall j \in N \lim _{\alpha_{j} \rightarrow 0} \frac{\lambda_{\min }^{j}\left(A_{j} y\right)}{\alpha_{j}}=+\infty \\
\Longrightarrow \quad \lim _{\alpha_{N} \rightarrow 0} \min _{j \leq r} \frac{\lambda_{\min }^{j}\left(A_{j} y\right)}{\alpha_{j}}=\min _{j \in B} \frac{\lambda_{\min }^{j}\left(A_{j} y\right)}{\alpha_{j}} .
\end{gathered}
$$

On the other hand, for $y \in S_{Y}$ such that $A_{N} y \notin K_{N}$,

$$
\lim _{\alpha_{N} \rightarrow 0} \min _{j \leq r} \frac{\lambda_{\min }^{j}\left(A_{j} y\right)}{\alpha_{j}}=-\infty .
$$


Finally, for $y \in S_{Y}$ such that $A_{N} y \in \partial K_{N}$, we have

$$
\lim _{\alpha_{N} \rightarrow 0} \min _{j \leq r} \frac{\lambda_{\min }^{j}\left(A_{j} y\right)}{\alpha_{j}}= \begin{cases}0 & \text { if } A_{B} y \in \operatorname{int}\left(K_{B}\right) \\ \min _{j \in B} \frac{\lambda_{\min }^{j}\left(A_{j} y\right)}{\alpha_{j}} & \text { otherwise. }\end{cases}
$$

Taking the maximum over $y \in S_{Y}$ on the equalities (19), (20), and (21) and using Lemma 2, it follows that

$$
\lim _{\alpha_{N} \rightarrow 0} \max _{y \in S_{Y}} \min _{j \leq r} \frac{\lambda_{\min }^{j}\left(A_{j} y\right)}{\alpha_{j}}=\max _{\substack{y \in S_{Y} \\ A_{N}}} \min _{j \in K_{N}} \frac{\lambda_{\min }^{j}\left(A_{j} y\right)}{\alpha_{j}} .
$$

This settles the left hand sides.

Now recall, the right hand side of the equality in Theorem 1 is

$$
\min _{\widetilde{A} \in \Sigma} \max _{j \leq r} \frac{\left\|A_{j}-\widetilde{A}_{j}\right\|}{\alpha_{j}} .
$$

Take $\widetilde{A} \in \Sigma$. Then

$$
\begin{aligned}
\widetilde{A}_{N} \neq A_{N} & \Longrightarrow \exists j \in N\left\|A_{j}-\widetilde{A}_{j}\right\| \neq 0 \\
& \Longrightarrow \quad \exists j \in N \lim _{\alpha_{j} \rightarrow 0} \frac{\left\|A_{j}-\widetilde{A}_{j}\right\|}{\alpha_{j}}=+\infty \\
& \Longrightarrow \lim _{\alpha_{N} \rightarrow 0} \max _{j \leq r} \frac{\left\|A_{j}-\widetilde{A}_{j}\right\|}{\alpha_{j}}=+\infty
\end{aligned}
$$

This implies that

$$
\lim _{\alpha_{N} \rightarrow 0} \min _{\widetilde{A} \in \Sigma} \max _{j \leq r} \frac{\left\|A_{j}-\widetilde{A}_{j}\right\|}{\alpha_{j}}=\lim _{\alpha_{N} \rightarrow 0} \min _{\substack{\widetilde{A} \in \Sigma \\ \widetilde{A}_{N}=A_{N}}} \max _{j \leq r} \frac{\left\|A_{j}-\widetilde{A}_{j}\right\|}{\alpha_{j}} .
$$

But if $\widetilde{A}_{N}=A_{N}$ then $\frac{\left\|A_{j}-\widetilde{A}_{j}\right\|}{\alpha_{j}}=0$ for all $j \in N$. Therefore,

$$
\lim _{\alpha_{N} \rightarrow 0} \min _{\substack{\widetilde{A} \in \Sigma \\ \widetilde{A}_{N}=A_{N}}} \max _{j \leq r} \frac{\left\|A_{j}-\widetilde{A}_{j}\right\|}{\alpha_{j}}=\lim _{\alpha_{N} \rightarrow 0} \min _{\substack{\widetilde{A} \in \Sigma \\ \widetilde{A}_{N}=A_{N}}} \max _{j \in B} \frac{\left\|A_{j}-\widetilde{A}_{j}\right\|}{\alpha_{j}}
$$

and thus the equality for the right hand sides. 


\section{References}

[1] F. Alizadeh and D. Goldfarb. Second-order cone programming. Math. Program., 95:3-51, 2003.

[2] J. Baez. The octonions. Bull. Amer. Math. Soc., 39:145-205, 2002.

[3] H.H. Bauschke, O. Güler, A.S. Lewis, and H.S. Sendov. Hyperbolic polynomials and convex analysis. Canad. J. Math., 53:470-488, 2001.

[4] J. Borwein and A. Lewis. Convex Analysis and Nonlinear Optimization: Theory and Examples. Springer-Verlag, 2000.

[5] H. Braun and M. Koecher. Jordan-Algebren. Number 128 in Grundlehren der mathematischen Wissenschaften. Springer, 1966.

[6] D. Cheung and F. Cucker. A new condition number for linear programming. Math. Program., 91:163-174, 2001.

[7] D. Cheung and F. Cucker. Probabilistic analysis of condition numbers for linear programming. Journal of Optimization Theory and Applications, 114:55-67, 2002.

[8] D. Cheung, F. Cucker, and R. Hauser. Tail decay and moment estimates of a condition number for random linear conic systems. SIAM Journal on Optimization, 15:1237-1261, 2005.

[9] D. Cheung, F. Cucker, and J. Peña. A condition number for second order conic systems. Preprint, 2006.

[10] J.H. Conway and D.A. Smith. On quaternions and octonions: Their geometry, arithmetic, and symmetry. A K Peters, Ltd., 2003.

[11] F. Cucker and J. Peña. A primal-dual algorithm for solving polyhedral conic systems with a finite-precision machine. SIAM Journal on Optimization, 12:522-554, 2002.

[12] F. Cucker and M. Wschebor. On the expected condition number of linear programming problems. Numer. Math., 94:419-478, 2002.

[13] T. Dray and C. Manogue. The exceptional Jordan eigenvalue problem. Internat. J. Theoret. Phys., 38:2901-2916, 1999.

[14] J. Dunagan, D.A. Spielman, and S.-H. Teng. Smoothed analysis of Renegar's condition number for linear programming. Preprint available at http://theory.lcs.mit.edu/ spielman, 2003.

[15] J. Faraut and A. Korányi. Analysis on Symmetric Cones. Clarendon Press, 1994.

[16] L. Faybusovich. Linear systems in Jordan algebras and primal-dual interiorpoint algorithms. Journal of Computational and Applied Mathematics, 86:149$175,1997$. 
[17] R.M. Freund and J.R. Vera. Condition-based complexity of convex optimization in conic linear form via the ellipsoid algorithm. SIAM Journal on Optimization, 10:155-176, 1999.

[18] R.M. Freund and J.R. Vera. Some characterizations and properties of the "distance to ill-posedness" and the condition measure of a conic linear system. Math. Program., 86:225-260, 1999.

[19] J.-L. Goffin. The relaxation method for solving systems of linear inequalities. Math. Oper. Res., 5:388-414, 1980.

[20] R. Hauser and T. Müller. Algebraic tail decay of condition numbers for random conic systems under a general family of input distributions. Preprint available at http://www.optimization-online.org/DB_HTML/2006/02/1336.html, 2006.

[21] S. Lang. Algebra. Addison-Wesley, 3rd edition, 1993.

[22] Yu.E. Nesterov and M.J. Todd. Self-scaled barriers and interior-point methods for convex programming. Math. Oper. Res., 22:1-42, 1997.

[23] Yu.E. Nesterov and M.J. Todd. Primal-dual interior-point methods for selfscaled cones. SIAM Journal on Optimization, 8:324-364, 1998.

[24] J. Renegar. Incorporating condition measures into the complexity theory of linear programming. SIAM Journal on Optimization, 5:506-524, 1995.

[25] J. Renegar. Linear programming, complexity theory and elementary functional analysis. Math. Program., 70:279-351, 1995.

[26] J. Renegar. A Mathematical View of Interior-Point Methods in Convex Optimization. SIAM, 2000.

[27] J. Renegar. Hyperbolic programs and their derivative relaxations. To appear at Found. Comput. Math., 2006.

[28] W. Rudin. Real and Complex Analysis. McGraw-Hill, 1987.

[29] H. Schaefer. Topological Vector Spaces. Springer-Verlag, 2nd edition, 1999.

[30] M. Sousa Lobo, L. Vandenberghe, S. Boyd, and H. Lebret. Applications of second-order cone programming. Linear Algebra and its Applications, 284:193$228,1998$. 